\section{SMGrøup}

SM Journal of

Nephrology and

Kidney Diseases

\title{
Monitoring Intracellular ( Vc), Interstitial (Vi) and Intravascular (Vb) Volume Changes During Dialysis (HD) in a Chronic Unit (CU) Compared to an Acute Unit (AU) Setting
}

Leslie D Montgomery ${ }^{1 \star}$, Richard W Montgomery ${ }^{1}$, Wayne A Gerth ${ }^{1}$, Susie Q Lew ${ }^{2}$, Manuel T Velasquez ${ }^{2}$, Julian M Stewart ${ }^{3}$, Marvin S Medow ${ }^{3}$ and Michael M Klein ${ }^{3}$ ${ }^{1}$ LDM Associates, San Jose, CA, USA

${ }^{2}$ The George Washington University School of Medicine and Health Sciences, Washington, DC, USA

${ }^{3}$ New York Medical College, Valhalla, NY, USA

\section{Abstract}

Introduction: End-staged renal disease (ESRD) patients may be dialyzed under both chronic and/or acute conditions. No information was found in the literature regarding the intracellular, interstitial and intravascular volume changes that take place under both chronic and acute dialysis.

Aim: To measure the volume changes of the intracellular $\left(V_{c}\right)$, interstitial $\left(V_{i}\right)$, and intravascular $\left(V_{b}\right)$ compartments during hemodialysis (HD) in end-stage renal disease patients in an outpatient or chronic unit (CU) and in an inpatient or acute unit (AU) setting and create pattern profiles for each treatment center.

Materials and methods: In a descriptive study, electrical impedance spectroscopy (EIS) was used to obtain noninvasive real time relative measurements of $V_{c}, V_{i}$, and $V_{b}$ changes continuously during dialysis with four electrodes place on the calf. A total of 70 ESRD patients was included in this study ( 23 chronic and 47 acute).

Results: The average pre-dialysis percentage volumes in the $\mathrm{V}_{b}, \mathrm{~V}_{i}$, and $\mathrm{V}_{c}$ compartments of the chronic patients was $51 \%, 19 \%$, and $29 \%$, respectively. The similar percentage volumes for the acute patients were $26 \%$, $29 \%$, and $45 \%$. The average post-dialysis volumes for the chronic patients were $49 \%, 15 \%$, and $36 \%$. The postdialysis values for the acute patients were $27 \%, 25 \%$, and $49 \% . V_{c}$ increased during hemodialysis in both patient populations. AU patients retained a larger percentage of total fluid in the $\mathrm{V}_{\mathrm{i}}$ and $\mathrm{V}_{\mathrm{c}}$ compartments.

Conclusion: The EIS technique was able to track fluid volume changes in the three principal fluid compartments of the calves of ESRD patients under both chronic and acute treatment. Results indicate that $\mathrm{V}_{c}$ in the monitored calves tended to increase during hemodialysis in both $\mathrm{CU}$ and $\mathrm{AU}$ patients as overall calf volumes were reduced.

\section{Introduction}

Chronic dialysis patients receive repeated treatments in an outpatient hemodialysis unit (Chronic). Their dialysis needs are often well known and predictable. Occasionally patients need emergency dialysis performed in the hospital or emergency department (Acute). Their treatment conditions may not be known to the medical staff. Compartment fluid volume removal data from patients during acute and chronic dialysis would be helpful in gaining insight into the specific hemodialysis operating characteristics needed under these conditions. Such volume from the intracellular $\left(\mathrm{V}_{\mathrm{c}}\right)$, interstitial $\left(\mathrm{V}_{\mathrm{i}}\right)$, and intravascular $\left(\mathrm{V}_{\mathrm{b}}\right)$ information does not seem to be available in the literature.

\section{Objective}

The aim of this study was to use electrical impedance spectroscopy (EIS) to measure $V_{c}, V_{i}$, and $\mathrm{V}_{\mathrm{b}}$ volume changes during hemodialysis (HD) in End-Stage Renal Disease ((ESRD) patients in an outpatient or Chronic Unit (CU) and in an inpatient or Acute Unit (AU) setting and create pattern profiles for each treatment center.

\section{Methods}

\section{Electrical Impedance Spectrograph Description}

The EIS (Z-Scan-2, U.F.I. Inc, Morro Bay, CA) used in this study, combines two bioimpedance instruments into a single device. It combines a fixed frequency Impedance Plethysmograph (IPG)

\section{ISSN: $2576-5450$}


and a multi-frequency electrical impedance spectrograph (EIS) into one unit. We use the IPG mode to quantify segmental blood flow and total segmental conductive volume. The EIS mode monitors segmental intracellular and extracellular compartment volumes similar to other EIS devices. However, our proprietary software also allows the Z-Scan-2 to divide the extracellular compartment volume into its intravascular and interstitial components.

The current Z-Scan-2 design, shown in Figure 1 measures subject tissue impedance, resistance, and reactance while "sweeping" across 40 discrete excitation frequencies over a wide frequency range when functioning as an EIS for compartment volume measurements [1]. Montgomery et al. reports the validation of the Z-Scan-2 [2].

\section{Specific Aims}

Our initial intended area of application specified the use of our device on all patients undergoing hemodialysis. We used information from two different data sources to demonstrate this capability. For the sake of seeing how our patient recordings track hematocrit we employed data obtained at a chronic dialysis center from "walk-in" patients during their routine dialysis sessions. For the sake of exploring how our data might provide added insight into the relationship between the progress of the dialysis session and difficulties experienced by the patient (e.g., fainting, cramps, etc.) we collected data from an acute treatment center. Patients treated in the acute unit were hospitalized and presented a rather wide range of symptoms during dialysis.

The protocols for patient tests at both centers were fully approved by their respective Institutional Internal Review Boards before any human testing was conducted.

The different patient populations at the chronic and acute centers provided an opportunity to exercise our protocols under conditions that will be encountered during different types of dialysis sessions.

Each patient at the chronic center was tested three times on subsequent days during a given week. Patients at the acute unit were generally hospitalized for a short period of time and were tested as their availability allowed. Most of these patients were therefore tested only once. In this way, the total number of chronic tests was 23 and the number of acute tests was 47 for a total from both centers of 70 patient tests.

\section{Subject Recruitment}

Inclusion/exclusion criteria and subject population demographics are described below.

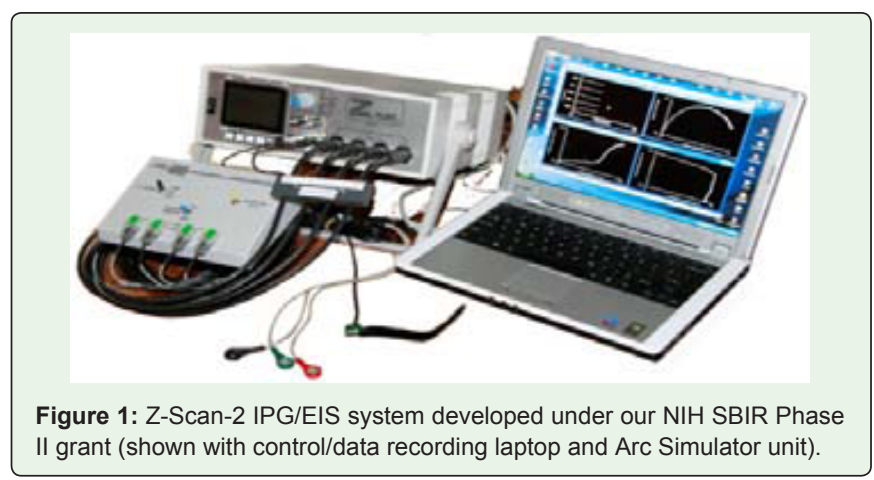

Inclusion criteria were ages over 21, patients with a well-charted history of ESRD, who regularly attended a chronic dialysis unit or were treated in an acute setting, were willing to undergo testing, and gave informed consent of the subject protocol.

Exclusion criteria were any of the following: arrhythmias, unstable angina, active gastrointestinal bleeding (for patient's safety reasons), pregnancy, peripheral vascular disease, psoriasis, peripheral neuropathy, liver failure, presence of tumors, collagen vascular disease, dermatologic problems, chronic angioedema or lymphedema. Implanted pacemaker or other implanted electrical device usage, uncontrolled hyper-thyroidism, chronic substance abuse, patients with surgical procedures on the leg may not be used for investigation, or any condition precluding the subject following the protocol.

\section{Protocol}

In the chronic dialysis unit, hemodialysis (HD) treatment was conducted according to the nephrologist's prescription. HD was performed using Polyflux Series (8L, $170 \mathrm{H}$ or $210 \mathrm{H}$ ) high flux dialyzers. (Hechingen, Germany) for 3 to 4 hours depending on the prescribed HD treatment by the patient's attending nephrologist. Testing was performed on each of three dialysis days during a single week for every subject. Patients in the chronic unit were seated in a recliner. Patients in the acute unit were treated in the supine position.

The Z-Scan-2 instrumentation was attached to the subject's dominant lower leg as shown in Figure 2. The source electrodes were placed just above the knee, and just above the lateral malleolus, the sampling electrodes were placed just below the knee and above the ankle.

During each HD treatment, body weight was measured before and at the end of the HD session. Systolic and diastolic BP (by the arm cuff method) and heart rate (HR) was measured every 30 min during the entire session. Signs and symptoms of hypovolemia, such as nausea, vomiting, dizziness, lightheadedness, thirst, fatigue, and cramps were also recorded.

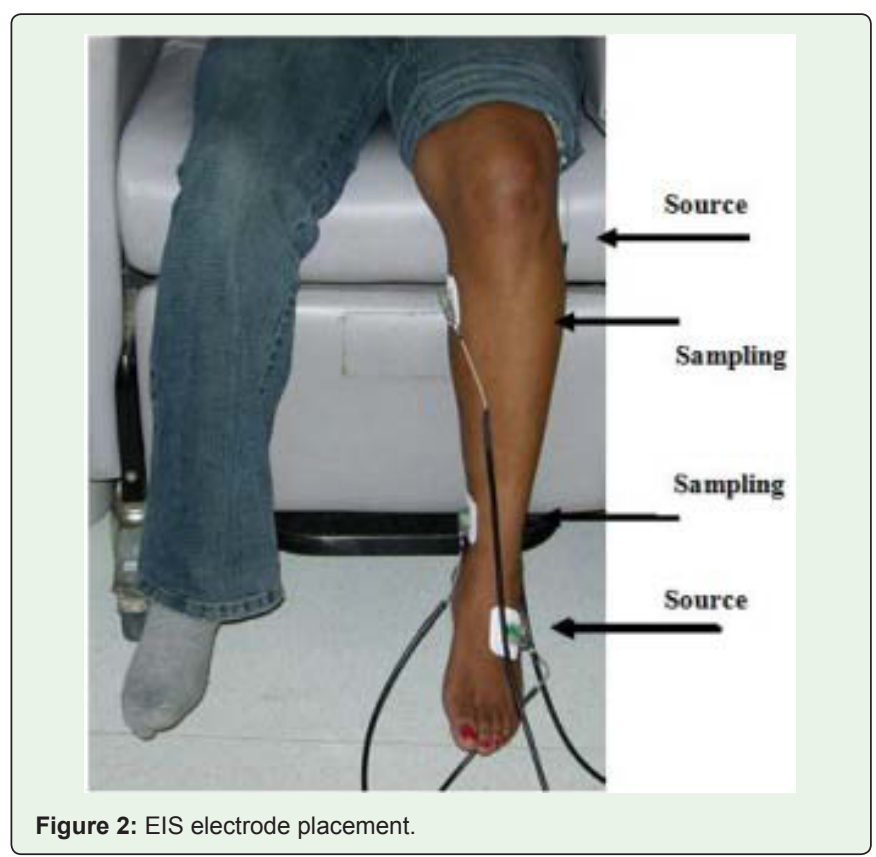




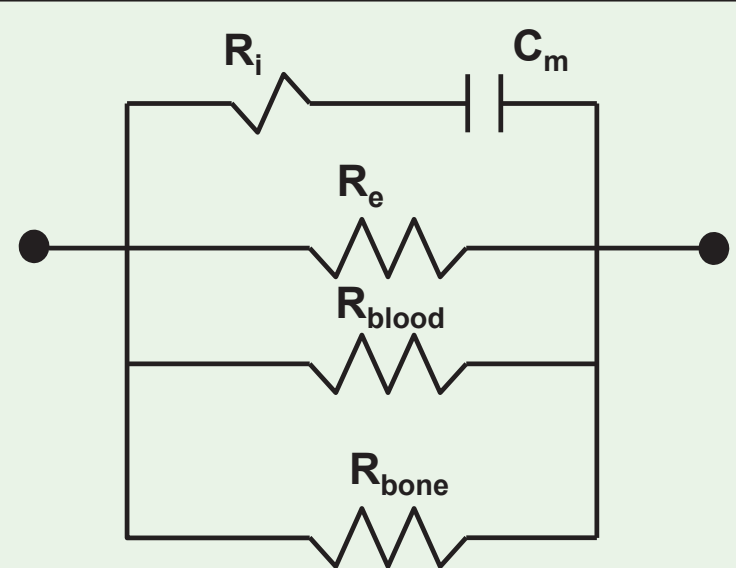

Figure 3: Equivalent circuit diagram used in EIS analysis for intracellular, interstitial, and intravascular volumes.

Following a 30-minute semi-reclined acclimatization/ instrumentation period the measurements listed above were recorded for a period of 30 minutes, which then served as the pre-dialysis control values. After the control measurements were completed, the patients underwent hemodialysis as per routine for a maximum period of 4 hours. All recordings were made during the period of dialysis and during the post-dialysis recovery period. Symptoms such as breathlessness, dizziness, presyncope, and signs such as blood pressure and heart rate were also recorded, as per dialysis-unit routine, during each test. We recorded changes in dialysate flow rate, blood flow rate and net fluid removal on a continuous basis.

\section{Anthropometric measurements}

Age, race, gender, body weight (in $\mathrm{kg}$ ), height (in $\mathrm{cm}$ ) and time since diagnosis of ESRD were used to characterize each patient undergoing dialysis.

\section{Data Analysis}

Various resistance/capacitance (R/C) models [3-6] are used to quantify segmental volumes from recorded impedance measurements. The $\mathrm{R} / \mathrm{C}$ model that is used by most currently available impedance systems represents the tissue as two parallel conductance paths: one through an extracellular compartment having average resistance (Re) and the other through an intracellular compartment having average resistance $(\mathrm{Ri})$ and capacitance $(\mathrm{Cm})$. This model, which is limited to the determination of "intracellular" and "extracellular" resistances ( $\mathrm{Ri}$ and $\mathrm{Re}$, respectively), can therefore only be used to estimate the corresponding intracellular, extracellular, and overall volumes of a monitored body segment. Unfortunately, an issue of principal concern in many clinical contexts and during dialysis is how the "extracellular volume" is distributed between its interstitial and

Table 1: Characteristics of chronic and acute dialysis centers.

\begin{tabular}{|c|c|c|}
\hline Source & Chronic & Acute \\
\hline Patients & Walk-in patients, in their usual state of health except for volume \\
overloaded. & Hospitalized, with a variety of symptoms. \\
\hline Repeatability & Routine repeated sessions for each patient. & Significant health changes between sessions. \\
\hline Interventions & $\begin{array}{c}\text { Nearly constant ultra filtration } \\
\text { rates; minor interventions (e.g., saline injections). }\end{array}$ & Frequent UF-rate changes; \\
\hline
\end{tabular}

Citation: Montgomery LD, Montgomery RW, Gerth WA, Lew SQ, Velasquez MT, Stewart JM, Medow MS, et al. Monitoring Intracellular $\left(\mathrm{V}_{\mathrm{c}}\right)$, Interstitial $\left(\mathrm{V}_{\mathrm{i}}\right)$ and Intravascular $\left(\mathrm{V}_{\mathrm{b}}\right)$ Volume Changes During Dialysis (HD) in a Chronic Unit (CU) Compared to an Acute Unit (AU) Setting. J Nephrol Kidney Dis. 2019; $3(1)$ : 1019. https://dx.doi.org/10.36876/smjnkd.1019 


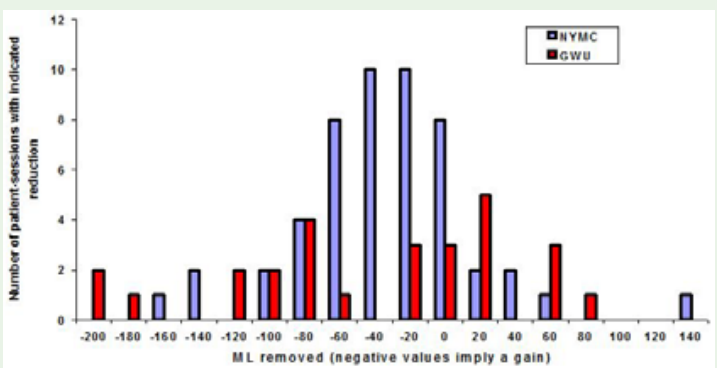

Figure 5A: Calf intracellular volume reduction - $\mathrm{ML}$ removed. NYMC = Chronic; GWU = Acute

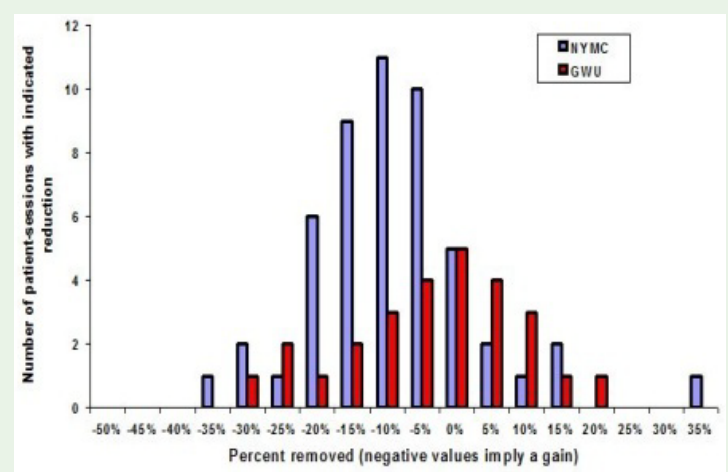

Figure 5B: Calf intracellular percent volume reduction

Variances within each data set, for each compartment are too large to make any claims regarding statistical significance of differences between the centers. (Standard deviations for the percentage changes are probably not meaningful, though we have reported them.) Yet it may, nonetheless, be interesting to view the differences in terms of histograms of the distributions, starting with that for intracellular $\left(\mathrm{V}_{\mathrm{c}}\right)$, then interstitial $\left(\mathrm{V}_{\mathrm{i}}\right)$, and finally intravascular fluid $\left(\mathrm{V}_{\mathrm{b}}\right)$.

Figures 5-7 show the number of patients at each center who lost or gained various amounts of fluid and the percentage of compartment volume changes in their calf during dialysis.

\section{Discussion}

The Z-Scan-2 monitoring system makes it possible to observe fluid volume changes in each of the three calf compartments. But perhaps more importantly, this means that it is possible to monitor shifts in fluid distribution between the compartments. This, of course, is what relates most directly to changes in hematocrit and vascular refilling-which are of particular concern for patient management Table 2.

Table 2: Dialysis Subject Tally.

\begin{tabular}{|c|c|c|c|c|c|c|c|c|c|}
\hline \multirow[t]{2}{*}{ Center } & \multicolumn{2}{|c|}{ Caucasian } & \multicolumn{2}{|c|}{ African American } & \multicolumn{2}{|c|}{ Hispanic } & \multicolumn{2}{|c|}{ Other } & \multirow[t]{2}{*}{ Total } \\
\hline & M & $\mathrm{F}$ & M & $\mathrm{F}$ & M & $\mathrm{F}$ & $\mathrm{F}$ & M & \\
\hline Acute & 6 & 3 & 22 & 15 & 1 & 0 & 0 & 0 & 47 \\
\hline Chronic & 15 & 2 & 2 & 1 & 1 & 0 & 2 & 0 & 23 \\
\hline Total & 21 & 5 & 24 & 16 & 2 & 0 & 2 & 0 & 70 \\
\hline
\end{tabular}

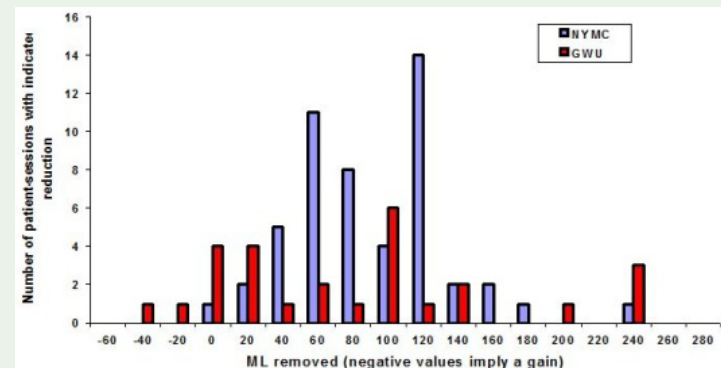

Figure 6A: Calf interstitial volume reduction - ML removed NYMC $=$ Chronic, $\mathrm{GWU}=$ Acute

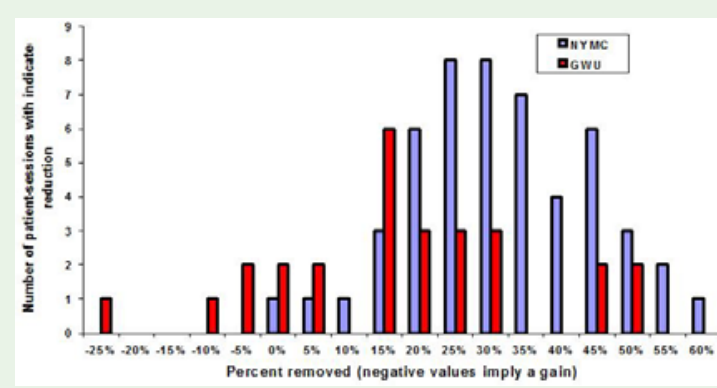

Figure 6B: Calf interstitial percent volume reduction.

Careful examination of Table 3 reveals many interesting differences between the two centers; for example, that the acute patients held a larger percentage of total fluid in the interstitial and intracellular compartments than did the chronic patients (both pre and post-dialysis). Also, for both centers, especially for chronic patients, intracellular fluid volume increased, as noted above Table 4.

Actual volumes of interstitial fluid removed from patients at the two centers were similar. However, the percentage distribution is shifted to the right for chronic patients because patients there presented with less interstitial fluid overload. Average pre-dialysis interstitial volumes were 270 milliliters at the chronic center compared with 425 milliliters at the acute center. Also, notice that two acute patients experienced an increase in interstitial fluid.

Of note, many patients at both centers (especially chronic ptients) experienced a gain in intracellular fluid (shown by the negative values on the horizontal axis. Montgomery et al. [15] discussed the potential intracellular volume increase that may take place during dialysis.

Table 3: Shows the average pre- and post-dialysis three compartment volumes and the associated percentage fluid distribution within the calves of the patients from the two centers Average Pre and post fluid Volumes and percentage fluid distributions in Calves.

\begin{tabular}{|l|c|c|c|c|c|c|}
\hline \multirow{2}{*}{} & \multicolumn{3}{|c|}{ CU } & \multicolumn{3}{c|}{ AU } \\
\cline { 2 - 7 } & $\mathbf{V}_{\mathrm{b}}$ & $\mathbf{V}_{\mathbf{i}}$ & $\mathbf{V}_{\mathbf{c}}$ & $\mathbf{V}_{\mathrm{b}}$ & $\mathbf{V}_{\mathbf{i}}$ & $\mathbf{V}_{\mathbf{c}}$ \\
\hline \multirow{2}{*}{ ML Pre } & 717 & 270 & 407 & 379 & 425 & 654 \\
\hline \multirow{2}{*}{ ML Post } & $51 \%$ & $19 \%$ & $29 \%$ & $26 \%$ & $29 \%$ & $45 \%$ \\
\hline & 621 & 189 & 451 & 383 & 353 & 705 \\
\hline & $49 \%$ & $15 \%$ & $36 \%$ & $27 \%$ & $25 \%$ & $49 \%$ \\
\hline
\end{tabular}




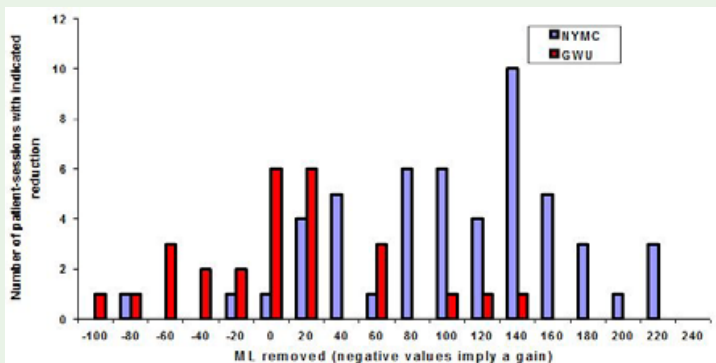

Figure 7A: Calf intravascular volume reduction - ML removed. NYMC = Chronic, GWU = Acute

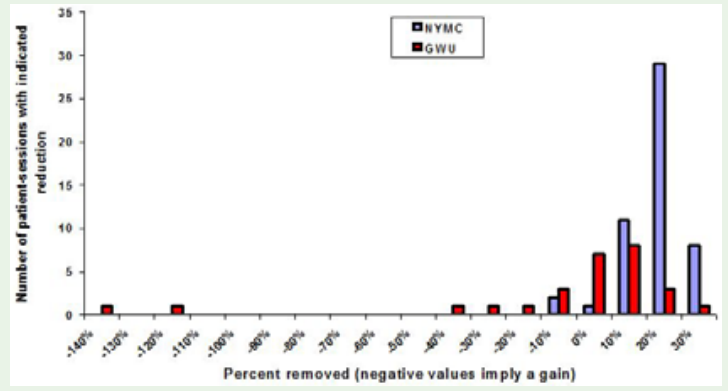

Figure 7B: Calf intravascular percent volume reduction.

Table 4: Shows the average volumes of fluid removed through dialysis from the three monitored calf compartments (and total calf, Vt) from the two centers. Minus signs indicate a garin rather than a reduction in fluid volume Average volumes of fluid removed from the calf through dialysis.

\begin{tabular}{|c|c|c|c|c|c|c|c|c|}
\hline Volumes removed & $\mathbf{V}_{b} \%$ & $\mathbf{V}_{\mathrm{i}} \%$ & $\mathrm{~V}_{\mathrm{c}} \%$ & $V_{t} \%$ & $\mathrm{~V}_{\mathrm{b}} \mathrm{ML}$ & $V_{i} M L$ & $\mathbf{V}_{c} \mathbf{M L}$ & $V_{t} M L$ \\
\hline \multicolumn{9}{|l|}{$C U(n=47)$} \\
\hline Mean & 12 & 30 & -11 & 9 & 96 & 81 & -44 & 133 \\
\hline std.Dev & 8 & 13 & 12 & 5 & 65 & 44 & 52 & 70 \\
\hline \multicolumn{9}{|l|}{$A U(n=23)$} \\
\hline Mean & -21 & 15 & -6 & 1 & -4 & 72 & -51 & 16 \\
\hline std.Dev & 73 & 20 & 13 & 4 & 58 & 81 & 81 & 46 \\
\hline
\end{tabular}

Figure 7B is distorted by the fact that two acute patients presented with extremely low vascular volume (fortunately, they gained) Acute patients on average presented with lower vascular volume, 379 milliliters, compared with 717 milliliters at the chronic center. The Figure 5A however, indicates a distinct distributional difference: All but three chronic patients experienced a reduction in vascular volume, usually large, while the acute patients experienced a much smaller reduction; several experienced a gain in vascular volume.

However, at this stage we regard such findings as merely an indication of the potential research use of the Z-Scan-2 monitoring system. We are reluctant to draw any causal inferences, since this was not designed to be a controlled experiment. There are too many differences between the two centers (e.g., different gender and ethnic distributions as well as the fact that one center treats walk-in patients and the other treats hospitalized patients).

Nonetheless, a well-designed research application of the Z-Scan-2 may reveal differences that might be important for patient management. For example, it may contribute to the debate addressed in the literature regarding the value of having walk-in patients lay down rather sit during dialysis.

\section{Summary}

1. Vc increased during hemodialysis in both patient populations.

2. The patterns of $\mathrm{V}_{c}$ changes differed between $\mathrm{CU}$ and $\mathrm{AU}$ patients.

3. AU patients retained a larger percentage of total fluid in the $\mathrm{V}_{\mathrm{i}}$ and $\mathrm{V}_{\mathrm{c}}$ compartments.

4. The distribution of $\mathrm{V}_{\mathrm{t}}$ response also differed between the two groups with $\mathrm{CU}$ patients showing greater volumes (implying greater amount of fluid removed) than AU patients.

\section{Conclusion}

The EIS technique was able to track fluid volume changes in the three principal fluid compartments of the calves of ESRD patients under both chronic and acute treatment. Results indicate that Vc in the monitored calves tended to increase during hemodialysis in both $\mathrm{CU}$ and $\mathrm{AU}$ patients as overall calf volumes were reduced.

\section{Funding}

This work was funded, in part, by NIH SBIR Grants 1 R43 HLO74524-01 and 2 R44 LO74524-02A2.s wish to thank Ms. Sharon Hanish, Brian Scholfield, and Marty Loughry of UFI, Inc., Morro Bay, CA for their technical support and encouragement during this project.

The authors from LDM Associates were the recipients of the National Heart, Lung and Blood Institute of the National Institutes of Health through SBIR Grants 1 R43 HL074524-01 and 2 R44 HL074524-02A2 entitled, "Intra/Extracellular Volume and Hemodynamics" between Sept. 1, 2003 and July 31, 2008.

\section{References}

1. Montgomery LD, Gerth WA, Montgomery RW, Lew SQ, Klein MM, Stewart $\mathrm{JM}$, et al. Monitoring intracellular, interstitial, and intravascular volume changes during fluid management procedures. Med Biol Eng Comput. 2013; 51: 1167-1175.

2. Montgomery LD, Montgomery RW, Gerth WA, Lew SQ, Velasquez MT. A system to monitor segmental intracellular, interstitial, and intravascular volume and circulatory changes during acute hemodialysis. J Electr Bioimp 2017; 8: 40-53

3. Ackman JJ, Seitz MA. Methods of complex impedance measurement in biologic tissue. CRC Critical Reviews in Biomedical Engineering 1984; 11: 281-311. 
4. Cole KS, Cole RH. Dispersion and absorption in dielectrics. J Chem Phys 1941; 9: 341-352.

5. Jindal GD. Impedance plethysmography for screening vascular disorders. J Postgrad Med. 1986; 32: 1-3.

6. Kanai $\mathrm{H}$, Katsuyuki S, Haeno M. Electrical measurement of fluid distribution in human legs: Estimation of extra- and intracellular fluid volume. J. Microwave Power 1983; 18: 233-243.

7. Bartok C, Schoeller DA. Estimation of segmental muscle volume by bioelectrical impedance spectroscopy. J Appl Physiol. 2004; 96: 161-166.

8. van der Sande FM, Luik AJ, Kooman JP, Verstappen V, Leunissen KML. Effect of intravenous fluids on blood pressure course during hemodialysis in hypotensive-prone patients. J Am Soc Nephrol. 2000; 11: 550-555.

9. Gerth WA, Montgomery LD, Wu YC. A computer-based bioelectrical impedance spectroscopic system for noninvasive assessment of compartmental fluid redistribution. In; Proceedings of Third Annual IEEE Symposium on Computer-Based Medical Systems. IEEE Computer Society Press, Chapel Hill, NC: 1990; 446-453.
10. Gerth WA, Watke CM. Electrical impedance spectroscopic monitoring of body compartment volume changes. J Clin Eng. 1993; 18: 253-260.

11. Sasser DC, Gerth WA, Wu YC. Monitoring of segmental intra- and extracellular volume changes using electrical impedance spectroscopy. J Appl Physiol. 1993; 74: 2180-2187.

12. Fricke $\mathrm{H}$. The electric capacity of suspensions of red corpuscles of a dog. Phys. Rev. 1925; 26: 682-687.

13. Fricke $\mathrm{H}$, Morse $\mathrm{S}$. The electric resistance and capacity of blood for frequencies between 800 and 4.5 million cycles. J Gen Physiol. 1925; 9: 153-167.

14. Fricke $\mathrm{H}$. The electric permittivity of a dilute suspension of membranecovered ellipsoids. J Appl Phys. 1953; 24: 644-646.

15. Montgomery LD, Montgomery RW, Gerth WA, Lew SQ, Klein MM, Stewart $\mathrm{JM}$, et al. Bioimpedance monitoring of cellular hydration during hemodialysis therapy. Hemodialysis Int. 2017; 21: 575-584. 